\title{
Optimal sequencing depth design for whole genome re-sequencing in pigs
}

\author{
Yifan Jiang ${ }^{1 \dagger}$, Yao Jiang ${ }^{1 \dagger}$, Sheng Wang ${ }^{1}$, Qin Zhang ${ }^{2}$ and Xiangdong Ding ${ }^{1 *}$
}

\begin{abstract}
Background: As whole-genome sequencing is becoming a routine technique, it is important to identify a costeffective depth of sequencing for such studies. However, the relationship between sequencing depth and biological results from the aspects of whole-genome coverage, variant discovery power and the quality of variants is unclear, especially in pigs. We sequenced the genomes of three Yorkshire boars at an approximately 20X depth on the Illumina HiSeq X Ten platform and downloaded whole-genome sequencing data for three Duroc and three Landrace pigs with an approximately 20X depth for each individual. Then, we downsampled the deep genome data by extracting twelve different proportions of $0.05,0.1,0.15,0.2,0.3,0.4,0.5,0.6,0.7,0.8$ and 0.9 paired reads from the original bam files to mimic the sequence data of the same individuals at sequencing depths of 1.09X, 2.18X, 3.26X, 4.35X, 6.53X, 8.70X, $10.88 X, 13.05 X, 15.22 X, 17.40 X, 19.57 X$ and $21.75 X$ to evaluate the influence of genome coverage, the variant discovery rate and genotyping accuracy as a function of sequencing depth. In addition, SNP chip data for Yorkshire pigs were used as a validation for the comparison of single-sample calling and multisample calling algorithms.

Results: Our results indicated that 10X is an ideal practical depth for achieving plateau coverage and discovering accurate variants, which achieved greater than $99 \%$ genome coverage. The number of false-positive variants was increased dramatically at a depth of less than $4 X$, which covered $95 \%$ of the whole genome. In addition, the comparison of multi- and single-sample calling showed that multisample calling was more sensitive than singlesample calling, especially at lower depths. The number of variants discovered under multisample calling was 13-fold and 2 -fold higher than that under single-sample calling at $1 X$ and $22 X$, respectively. A large difference was observed when the depth was less than 4.38X. However, more false-positive variants were detected under multisample calling.

Conclusions: Our research will inform important study design decisions regarding whole-genome sequencing depth. Our results will be helpful for choosing the appropriate depth to achieve the same power for studies performed under limited budgets.
\end{abstract}

Keywords: Genome coverage, Sequencing depth, Pig, Whole-genome sequencing

\section{Background}

Sequencing technologies have been widely used in many fields, such as human medicine [1], evolutionary chemistry [2-5], microbial ecology [6], agriculture [7] and animal breeding [8]. In sequencing, a key consideration is the sequencing depth, which is defined as the ratio of the total number of bases obtained by sequencing to the size of the genome or the average number of times each

\footnotetext{
* Correspondence: xding@cau.edu.cn

${ }^{+}$Yifan Jiang and Yao Jiang contributed equally to this work.

${ }^{1}$ National Engineering Laboratory for Animal Breeding, Laboratory of Animal Genetics, Breeding and Reproduction, Ministry of Agriculture, College of Animal Science and Technology, China Agricultural University, Beijing 100193, China

Full list of author information is available at the end of the article
}

base is measured in the genome [9]. Sequencing cost is the main concern in practice, which is mainly influenced by the sequencing depth, sequencing technology and sample size. Although the sequencing cost for a particular sample has decreased significantly in recent years, it is still a great burden for large-scale applications.

Sequencing depth has a great impact not only on sequencing cost but also on the biological results of sequencing data processing, e.g., the genomic assembly completeness and accuracy of a de novo assembly [10], the number of detected genes and expression levels in RNA-Seq [11], the proportion of rare variants and SNVs detected [12], and the accuracy of SNP calling and genotyping in whole-genome sequencing [13]. Therefore, it is

(C) The Author(s). 2019 Open Access This article is distributed under the terms of the Creative Commons Attribution 4.0 International License (http://creativecommons.org/licenses/by/4.0/), which permits unrestricted use, distribution, and 
particularly important to investigate sequencing depth to achieve a higher accuracy at a lower cost and to identify trade-offs between sequencing data quality and quantity.

Recently, there have been many studies on the impact of sequencing depth in RNA-Seq that have aimed to find the optimal sequencing depth for either de novo transcriptome assembly through the comparison of different nonmodel animals [14] or the study of gene expression in RNA-Seq [11]. In addition, similar plant studies have been carried out to investigate the optimal transcriptome coverage in Hevea brasiliensis [15]. For DNA sequencing, the research on sequencing depth has mainly focused on de novo genome assembly $[10,16]$ and genetic association studies of complex traits $[17,18]$. Additionally, several studies have explored the recommended coverage for reducing indel calling errors [19] and detecting copy number variations [20] in sequencing data. The impact of sequencing depth on single-cell sequencing has also been explored recently [21, 22]. However, most of the studies on sequencing depth conducted thus far have focused on simulated data $[18,23]$ or real datasets from humans [13] or pilot animals with small genomes, such as E. coli, S. kudriavzevii and C. elegans [10]; a limitation of simulated data is that mismapping around short indels cannot be taken into account [17], and these data cannot fully mimic the real situation in sequencing. Such investigations have seldom been conducted on animals with large genomes, such as pigs, and this research is useful not only for humans but also for livestock and other mammals.

In this study, we sequenced the whole genomes of three Yorkshire boars at a sequencing depth of approximately 20X. In addition, whole-genome sequencing data from three Landrace and three Duroc boars were downloaded from the NCBI SRA database to explore the relationship between sequencing depth and biological results from the aspects of whole-genome coverage, the variant discovery power and the quality of variants.

\section{Methods}

\section{Animal ethics statement}

Necessary permission was obtained from the owner of the farm for collecting the samples and using in the next study. All animal management and sample collection procedures strictly followed the protocol approved by the Institutional Animal Care and Use Ethics Committee (IACUC) at the China Agriculture University. And the IACUC of the China Agricultural University specifically approved this study (Permit Number: DK996).

\section{Whole-genome resequencing}

Blood samples from three Yorkshire boars were collected from a breeding farm in Beijing. Genomic DNA was extracted from the blood samples by using a
TIANamp Blood DNA spin kit (DP348; Tiangen, Beijing) following the manufacturer's protocol. The quality of all DNA samples was evaluated by agarose gel electrophoresis, and accurate quantification of DNA concentrations was conducted with a Qubit 2.0 fluorometer. Wholegenome sequencing was performed using the Illumina HiSeq X Ten platform according to the manufacturer's standard protocols and produced 150-bp paired-end reads in fastq format. Whole-genome sequencing data for three Duroc and three Landrace pigs in sra format were downloaded from the NCBI SRR database and then converted to fastq format by fastq-dump in the SRA Toolkit. All individuals that we selected were unrelated to each other.

\section{Sequencing quality control and NGS data processing}

To avoid reads with artificial bias, quality control was conducted by using the NGS QC Toolkit [24]. First, IlluQC.pl with the default parameters was used to remove reads that contained more than 30\% low-quality (quality value $\leq 20$ ) bases. Second, TrimmingReads.pl was used to trim the $3^{\prime}$ end of fragments. Then, highquality paired-end reads were mapped to the pig reference genome sequence (Sscrofa11.1 http://hgdownload. soe.ucsc.edu/goldenPath/susScr11/bigZips/) by the BWA (Burrows-Wheeler Aligner) [25] with the command 'bwa men $-\mathrm{M}-\mathrm{R}$ '.

\section{SNP calling and filtering}

The Genome Analysis Toolkit (GATK) [26] (version: 3.7) was used to call SNPs, following GATK best practices [27], in which realignment and recalibration were included. SNP calling and genotyping were performed by UnifiedGenotyper in GATK. Only SNPs on autosomes were used for the following analysis. Before SNP calling, Picard SortSam and Picard MarkDuplicates (http://broadinstitute.github.io/picard/) were used to sort and mark potential PCR duplicates separately. After SNP calling, hard filters were used to remove potential falsepositive SNPs and InDels. For SNPs, the following criteria were used for filtering, as suggested by the GATK documentation: "QD $<2.0 \|$ FS $>60.0\|\mathrm{MQ}<40.0\|$ HaplotypeScore $>13.0||$ MQRankSum $<-12.5 \|$ ReadPosRankSum $<-8.0$ ". For InDels, the criteria "QD $<2.0$ || FS > 200.0 || ReadPosRankSum < -20.0" were used for filtering, also suggested by the GATK documentation. Both single-sample calling and multisample calling (three samples from each breed) implemented in the GATK UnifiedGenotyper were used in our analysis.

\section{Relationships}

Principal component analysis (PCA) was performed via GCTA(a tool for Genome-wide Complex Trait Analysis) $[28,29]$ including all common SNPs in all individuals after filtering by the minor allele frequency $(\mathrm{MAF} \geq 0.05)$ 
and LD pruned (--indep-pairwise 10050 0.5). The heatmap of the genomic relationship between each individual was plotted by using a heatmap in $\mathrm{R}$.

Construction of samples with different sequencing depths Picard DownsampleSam was used to randomly downsample a bam file to construct different lower-depth samples. In this way, mate-pair reads were either kept or both discarded. Proportions of $0.05,0.1,0.15,0.2,0.3$, $0.4,0.5,0.6,0.7,0.8$ and 0.9 were set in a chained strategy for the raw mapped bam file, and average depths of $1.09 \mathrm{X}, 2.18 \mathrm{X}, 3.26 \mathrm{X}, 4.35 \mathrm{X}, 6.53 \mathrm{X}, 8.70 \mathrm{X}, 10.88 \mathrm{X}$, 13.05X, 15.22X, 17.40X, 19.57X and 21.75X corresponding to each proportion were produced. Together with the original greatest depth for each individual, a total of 12 gradient depths for each sample were used for further analysis. Figure 1 illustrates the workflow of nextgeneration sequencing (NGS) data processing.

\section{Variant annotation}

ANNOVAR [30] was used to annotate the variants with the dbSNP database. Following Ai et al. [4], those variants that met one of the following criteria were defined as potential loss-of-function (LoF) variants: (1) a SNP or small Indel within a coding region causing a frameshift of the open reading frame (ORF); (2) a SNP or small indel at a splice site; and (3) a SNP or small indel within a coding region resulting in a stopgain or stoploss.

\section{Comparison of data with different depths}

After variant calling and filtering, we compared data with different depths from the following aspects: (1) whole-genome coverage; (2) the number of SNPs discovered; (3) the discovery power for variants; and (4) the quality of variants evaluated by the novel rate and transition/transversion ratio.

The discovery power for each subsample is the ratio of variants in downsampled data to that in the original deep sequencing data.

The novel rate represents the proportion of variants not available in the dbSNP database to the total variants, as we defined the variants that were not included in the dbSNP database as novel variants. Here, we used the pig dbSNP database (Build ID: 150).

The transition/transversion ratio $(\mathrm{Ti} / \mathrm{Tv}$ ratio) is the proportion of the variants observed as transitions (between purines, or between pyrimidines) versus transversions (between purines and pyrimidines). The $\mathrm{Ti} / \mathrm{Tv}$ ratio is particularly useful for assessing the quality of single nucleotide polymorphisms inferred from sequencing data [31, 32]. A higher ratio generally indicates higher accuracy [27].

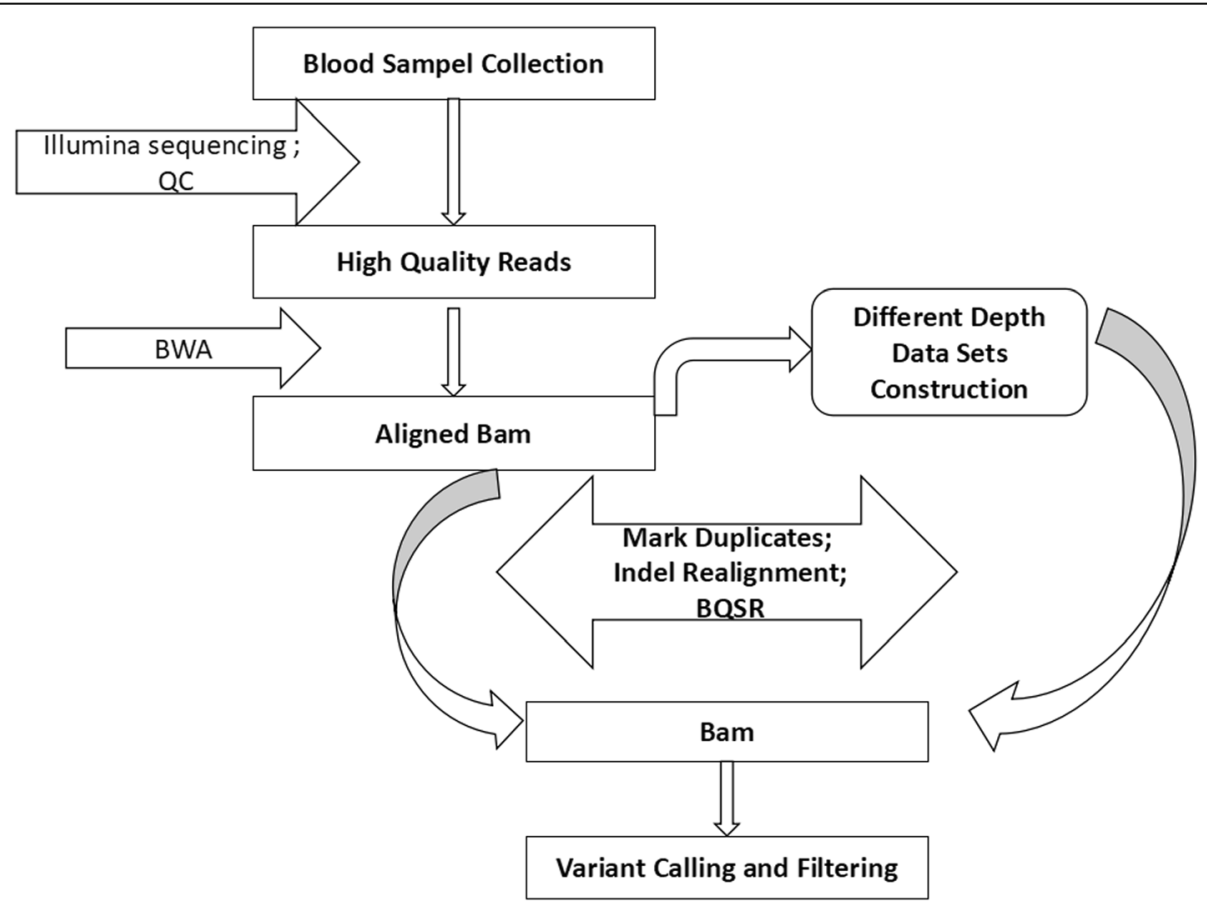

Fig. 1 Data processing pipeline. Our pipeline was identical for each sample. Original aligned bam files were mapped with clean fastq data. Then, we extracted different proportions of paired reads randomly from the original bam files to build samples with different depths. Markduplicates, Indel realignment and Base recalibration were applied for all bam files and the same procedures were used for SNP calling and filtering 
GATK DepthOfCoverage was used to compute the whole-genome coverage, and GATK VariantEval and R [33] were used to extract summary statistics from the results and for further analysis.

\section{BeadChip validation}

To further evaluate the accuracy of SNP calling, all samples of Yorkshire boars were genotyped with the PorcineSNP80K BeadChip (Illumina, San Diego, CA, USA), including 68,528 SNPs covering the whole genome. Genotype quality control was carried out with PLINK1.9 [34], in which SNPs with call rates less than 95\% and nonchromosomal SNPs were excluded. The common sites between each subdepth and beadchip dataset were counted, which were defined as common sites on the beadchip. The discordance rate for each sample and genotype caller was calculated for singlesample calling, which was defined as the fraction of the number of common sites for the sample between the PorcineSNP80K BeadChip and the whole-genome sequencing data processed by the genotype caller according to the number of genotypes that mismatched the chip genotype. For comparison between single-sample calling and multisample calling, the discordance rate for multisample calling was calculated, defined as the proportion of the number of genotype discordance sites to the common sites of the sample between the PorcineSNP80K BeadChip and whole-genome sequencing SNPs containing at least one nonreference allele.

\section{Results}

Summary of whole-genome sequencing and subsampling A summary of the sequencing data for three Yorkshire boars, three Duroc boars and three Landrace boars is shown in Table 1. The sequence data for each individual reached a depth of greater than 20-fold and covered more than $99 \%$ of the whole genome. Furthermore, multisample calling of the three individuals of each breed was implemented to perform a comparison of the SNP differences between the Duroc, Landrace and Yorkshire pigs, as shown in Table 2. A total of 10.26, 12.24, and 12.89 million variants were discovered for the Duroc, Landrace and Yorkshire pigs, respectively. Figure 2 shows the overlap of the variants discovered in each breed. A total of 5.57, 5.76, and 7.42 million common variants were discovered between Duroc-Landrace, Duroc-Yorkshire, and Landrace-Yorkshire, respectively, and 3.98 million common variants were discovered in all three breeds. Among the three pig breeds, Duroc presented the smallest number of SNPs, which was not surprising because the Sus scrofa reference genome comes from the Duroc breed. In addition, as shown in Table 2, when the variants were compared to the pig dbSNP database, a total of 8.67, 10.47 and 11.21 million variants were included in the dbSNP database, accounting for $84.50,85.53$ and $86.99 \%$ of the total variants of Duroc, Landrace and Yorkshire, respectively. ANNOVA annotation revealed $68,313,78,968$ and 85,372 variants in exon regions, among which there were $28,193,31,980$ and 33,538 loss-of-function (LoF) variants in Duroc, Landrace and Yorkshire, respectively. The transition/ transversion ratios for Duroc, Landrace and Yorkshire were $2.24,2.25$ and 2.34 , respectively. The detailed information for each individual is shown in Table 3.

The population structure was demonstrated through principal component analysis (PCA) (Fig. 3a) using 1.37 billion SNPs. Figure 3a shows that the Duroc, Yorkshire and Landrace breeds were distinctly separated from each other. The heatmap of the genomic relationships between each individual shown in Fig. $3 \mathrm{~b}$ indicated that the three individuals of each breed were clustered together and they were unrelated individuals to each other.

\section{The impact of depth on the coverage}

The coverage of the genome at each depth is illustrated in Fig. 4. All of the individuals from the three pig breeds presented almost the same trend, as the curves for all individuals nearly completely overlapped, and the coverage of each individual at a given depth was nearly the same. These results indicated that there was no breed or

Table 1 Summary statistics for the whole genome sequencing of data

\begin{tabular}{lllllll}
\hline Sample & Depth & Coverage & Map Ratio & Breed & Sex & SRA project \\
\hline S403505 & 24.40 & $99.41 \%$ & $99.30 \%$ & Yorkshire & male & male \\
S474607 & 22.31 & $99.39 \%$ & $99.53 \%$ & Yorkshire & male & male \\
S494203 & 21.79 & $99.32 \%$ & $98.76 \%$ & Yorkshire & PRJNA343658 \\
SAMN05791661 & 22.65 & $99.44 \%$ & $99.50 \%$ & Duroc & male & PRJNA343658 \\
SAMN05791665 & 21.72 & $99.46 \%$ & $99.57 \%$ & Duroc & male & PRJNA343658 \\
SAMN05791663 & 23.90 & $99.57 \%$ & $99.50 \%$ & Duroc & male & PRJNA343658 \\
SAMN05791650 & 19.89 & $99.30 \%$ & $99.47 \%$ & Landrace & male & PRJNA343658 \\
SAMN05791651 & 22.56 & $99.39 \%$ & $99.57 \%$ & Landrace & male & PRJNA343658 \\
SAMN05791660 & 19.36 & $99.38 \%$ & $99.73 \%$ & & &
\end{tabular}


Table 2 Summary statistics of variants discovered for three pig breeds, Duroc, Landrace and Yorkshire

\begin{tabular}{lllllll}
\hline Sample & Mean Depth & Total Variants & \%variants in dbSNP & Ti/Tv Ratio & Exons & LoF \\
\hline 3DD-sample & 22.76 & $10,260,949$ & 84.50 & 2.24 & 68,313 & 28,193 \\
3LL-sample & 20.60 & $12,239,211$ & 85.53 & 2.25 & 78,968 & 31,980 \\
3YY-sample & 21.88 & $12,887,321$ & 86.99 & 2.34 & 85,372 & 33,538
\end{tabular}

Mean Depth the sequencing depth for each breed on average, TotalVariants the number of SNPs discovered for each breed by 3 sample multisample calling, \%variants in dbSNP the number of SNPs in the dbSNP database, Ti/TV Ratio the proportion of the SNPs observed as transitions (between purines or between pyrimidines) versus transversions (between purines and pyrimidines), Exons the number of SNPs in exons, LoF the number of loss-of-function variants

individual specificity for the relationship of coverage and sequencing depth. In general, the coverage increased with the depth, but not linearly; there was a rapid increase in coverage from 61.17 to $95.42 \%$ when the depth was increased from $1.08 \mathrm{X}$ to $4.35 \mathrm{X}$, after which the coverage increased relatively slowly from 95.42 to $99 \%$ when the depth was increased from $5 \mathrm{X}$ to $10 \mathrm{X}$ and plateaued at a depth of 10X (at a proportion of 0.5 on average), which covered $\sim 99 \%$ of the whole genome and was very close to the coverage achieved at depths of $13.05 \mathrm{X}$, 15.22X, 17.40X, 19.57X and 21.75X of 99.21, 99.29, $99.34,99.37$ and $99.40 \%$ of the whole genome, respectively (Fig. 4). According to the curve presented in Fig. 4, a depth of $4.35 \mathrm{X}$ (at a proportion of 0.2 on average) was the inflection point, at which the coverage increased exponentially $(<4.35 \mathrm{X})$ and the increase ratio then decreased slightly. At the inflection point, $\sim 95.42 \%$ of the genome was covered.

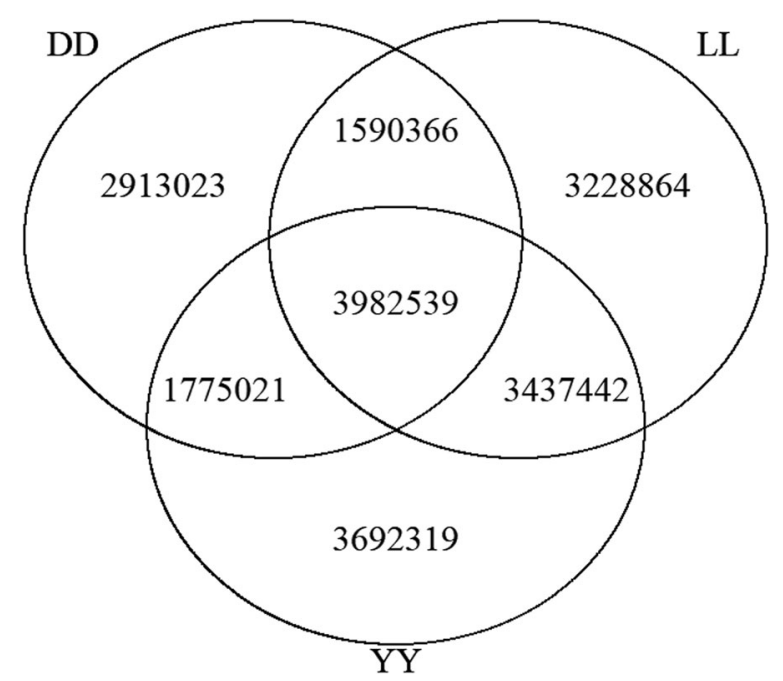

Fig. 2 The Venn diagram shows the variants discovered in each breed. The Venn diagram shows the overlap of the variants discovered in each breed. A total of 10,260,949, 12,239,211 and $12,887,321$ SNPs were discovered in Duroc, Landrace and Yorkshire, respectively. A total of 3,982,539 common variants were discovered in all three breeds, while 5,572,905, 5,757,560 and 7,419,981 common variants were discovered between Duroc-Landrace, Duroc-Yorkshire, and Landrace-Yorkshire, respectively

\section{Discovery of variants and quality of variants}

The total variants discovered at each depth for each sample are shown in Fig. 5a. The largest number of variants was discovered in Yorkshire, followed by Landrace and Duroc, similar to the results in Table 1, and the total variants for individuals within the same breed varied as well. The discovery power for all individuals (the proportion of variants in a downsampled individual to the greatest depth for the same individual) was also slightly different across breeds (Fig. 5b). The discovery power for variants exhibited a similar tendency to genome coverage, as shown in Fig. 4, which increased with depth, and a similar tendency was demonstrated across individuals. As expected, the number of variants discovered increased rapidly when the depth was less than $10 \mathrm{X}$, which was the point at which the coverage plateaued, after which the rate of increase slowed. At a depth of 10X, approximately 4.62 million SNPs were detected, accounting for $84.42 \%$ of the variants obtained from the deepest genome (21.75X in this study). The variant discovery power was increased by $15.58 \%$ on average when the depth was increased from $10.88 \mathrm{X}$ to $21.75 \mathrm{X}$, but it increased by $80.40 \%$ on average when the depth was increased from 1.09X to $10.88 \mathrm{X}$, accounting for 4.02 and $84.42 \%$ of the variants, respectively. At $4.35 \mathrm{X}$, which was the inflection point of the function of coverage with depth, 45.36\% (2.55 million) SNPs were detected.

The accuracy of SNP calling and genotyping was evaluated based on the novel rate and the transition/transversion ratio, as illustrated in Fig. 5c and Fig. 5d. The novel rate decreased with increasing sequencing depth (Fig. 5c), showing that the greater the depth of sequencing, the greater the number of reliable variants discovered. However, a large differences were observed between Duroc, Yorkshire, and Landrace. The sharpest decrease in the novel rate occurred in Yorkshire when the depth increased from $1.10 \mathrm{X}$ to $4.38 \mathrm{X}$, accounting for 16.99 and $13.45 \%$ of the variants in the dbSNP database, respectively, and then remaining basically unchanged, with a ratio of $\sim 13.2 \%$, when the depth was greater than $4.38 \mathrm{X}$, which may indicate that more false-positive variants were discovered when the depth was less than 4.38X. However, for Duroc and Yorkshire, the decline in 
Table 3 Summary statistics of variants discovered for each individual

\begin{tabular}{|c|c|c|c|c|c|c|}
\hline Breed & Sample & Total Variants & \%variants in dbSNP & Ti/Tv Ratio & Exons & LoF \\
\hline $\mathrm{DD}$ & SAMN05791661 & $4,361,699$ & 83.84 & 2.18 & 28,321 & 11,783 \\
\hline DD & SAMN05791665 & $4,720,118$ & 84.00 & 2.20 & 31,091 & 13,272 \\
\hline DD & SAMN05791663 & $4,761,804$ & 85.38 & 2.26 & 29,916 & 11,992 \\
\hline LL & SAMN05791650 & $4,664,664$ & 84.42 & 2.16 & 29,287 & 12,430 \\
\hline $\mathrm{LL}$ & SAMN05791651 & $5,255,293$ & 85.29 & 2.21 & 33,575 & 13,768 \\
\hline $\mathrm{LL}$ & SAMN05791660 & $5,433,413$ & 86.27 & 2.25 & 35,521 & 14,111 \\
\hline$Y Y$ & S494203 & $5,730,270$ & 86.99 & 2.31 & 39,094 & 15,304 \\
\hline YY & S409308 & $5,528,070$ & 86.74 & 2.30 & 38,986 & 15,630 \\
\hline YY & S474607 & $6,047,346$ & 86.81 & 2.28 & 38,828 & 15,482 \\
\hline
\end{tabular}

TotalVariants the number of SNPs discovered for each individual by single-sample calling, \%variants in the dbSNP databbase the number of SNPs in the dbSNP database, Ti/TV Ratio the proportion of the SNPs observed as transitions (between purines, or between pyrimidines) to transversions (between purines and pyrimidines), Exons the number of SNPs on exons, LoF the number of loss-of-function variants

the novel rate was slow when the depth was increased from $1 \mathrm{X}$ to $22 \mathrm{X}$.

$\mathrm{The} \mathrm{Ti} / \mathrm{Tv}$ ratios for each depth in all samples ranged from 1.99 to 2.34 .

as shown in Fig. 5d; Yorkshires presented higher Ti/Tv ratios than Durocs and Landraces, and the $\mathrm{Ti} / \mathrm{Tv}$ ratios for Durocs and Landraces were similar. In general, the variation of the $\mathrm{Ti} / \mathrm{Tv}$ ratio was not large; when the sequencing depth was increased from $\sim 1 \mathrm{X}$ to $\sim 21 \mathrm{X}$, the ranges of the $\mathrm{Ti} / \mathrm{Tv}$ ratio were $2.00 \sim 2.21,2.01 \sim 2.21$, and 2.31 2.30 for Duroc, Yorkshire and Landrace, respectively. Only the $\mathrm{Ti} / \mathrm{Tv}$ ratios for Duroc and Landrace increased with depth, indicating higher accuracy of SNP calling. However, the Ti/Tv ratio basically remained unchanged for Yorkshire.

\section{BeadChip validation}

A total of 56,963 SNPs from the PorcineSNP80K BeadChip data remained after quality control. Figure 6 shows the common sites between each depth and beadchip dataset. These results showed that more common variants were discovered as the depth increased, and multisample calling revealed more variants than single-sample calling both for all variants

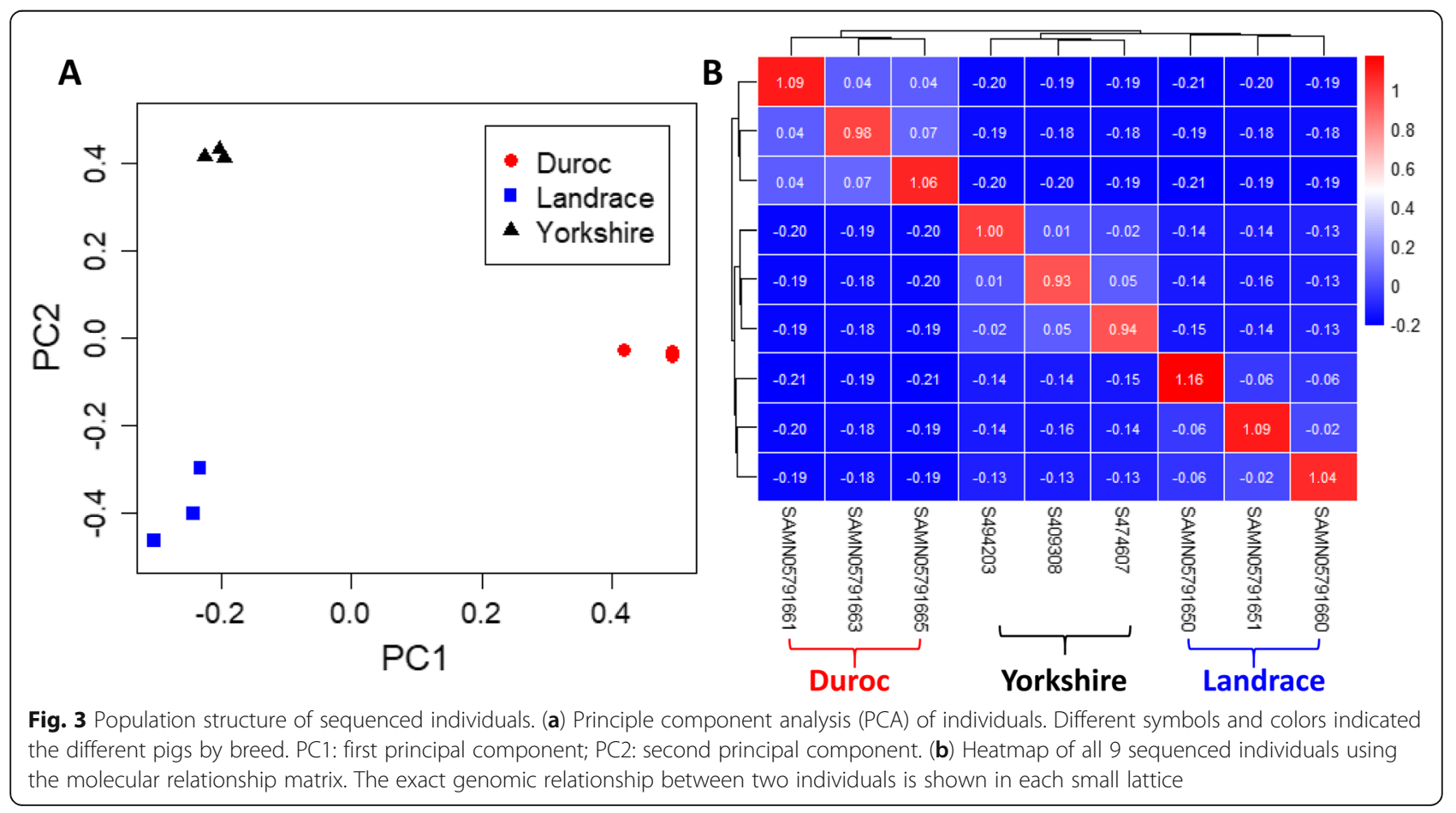




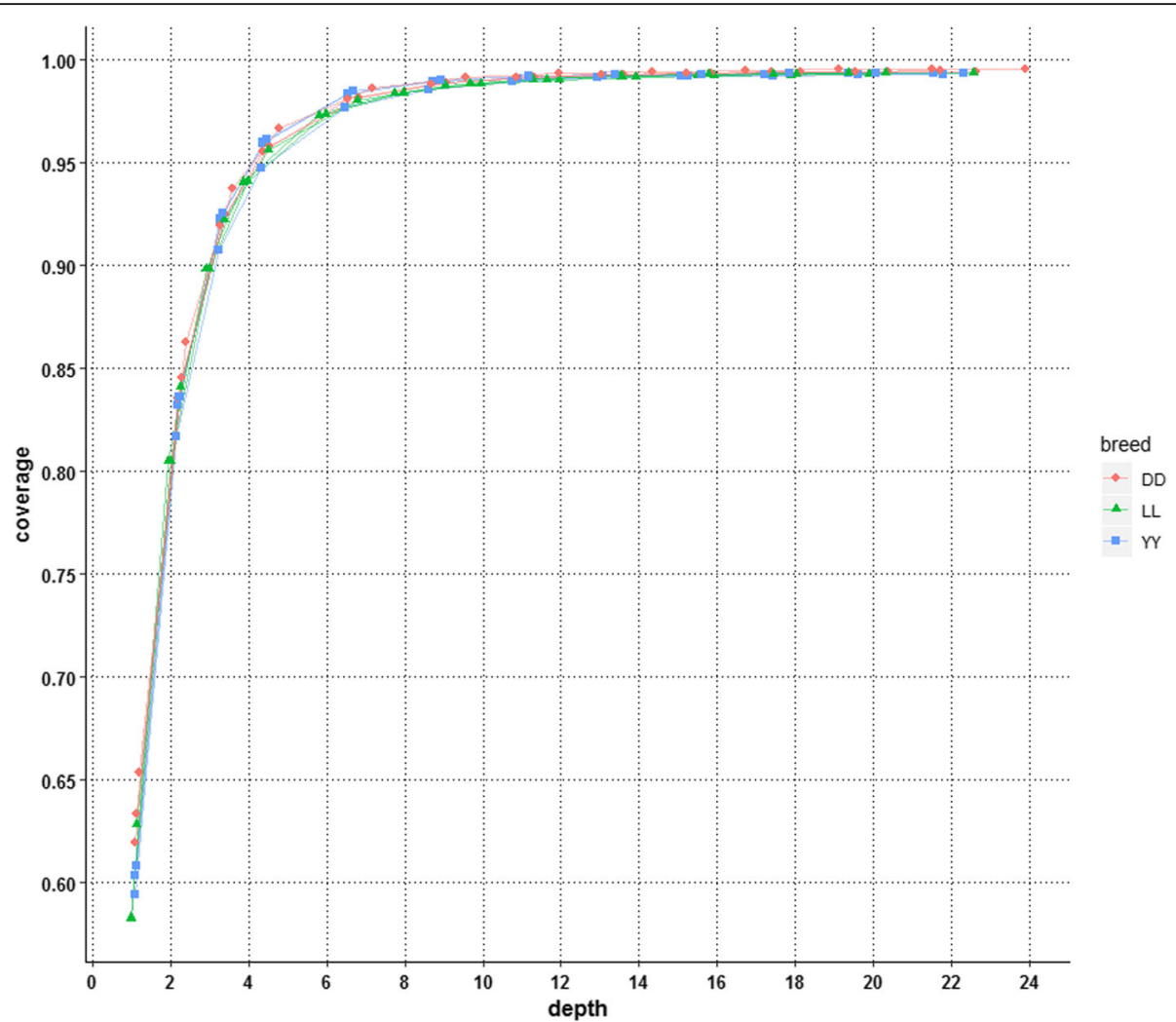

Fig. 4 Coverage with sequencing depth for each downsampled genome. Whole-genome coverage as a function of sequencing depth for each downsampled genome

and for only variants with at least one nonreference allele considered. The numbers of variants discovered via multisample calling were 2 times and 1.5 times greater than those obtained via single-sample calling at the greatest depth when all variant sites or only alternative reference sites were considered, respectively. The difference of the total variants discovered for multisample calling compared to single-sample calling increased as the sequencing depth decreased, as shown in Fig. 7a, especially when the depth was less than $4.38 \mathrm{X}$.

Figure $7 \mathrm{~b}$ shows the discordance rate with the SNP panel for Yorkshire under two scenarios of multisample and single-sample calling strategies. As expected, the discordance rate decreased with increasing depth. The two SNP calling strategies showed different performances. For single-sample calling, the discordance rate decreased slightly to $3.22 \%$ from $4.48 \%$ when the depth was increased from $1.10 \mathrm{X}$ to $21.88 \mathrm{X}$, while multisample calling yielded a much higher discordance rate of $27.16 \%$ at a depth of $1.10 \mathrm{X}$, which sharply decreased with increasing depth, then stabilized, with the discordance rate reaching $2.96 \%$ at a depth of 21.88X.

\section{Discussion}

In this study, we aimed to provide a comprehensive understanding of the relationship between data quality and the quantity of SNP calling and genotyping in pig whole-genome sequencing. Three popular pig breeds, Duroc, Yorkshire and Landrace, were examined, and three boars from each breed were sequenced at approximately 20X depth. To the best of our knowledge, there have been no similar studies in other livestock, such as cattle or poultry. Our findings can therefore serve as a general guide for researchers to choose an optimal sequencing depth. We extracted paired read randomly from the original bam files at proportions of 0.05, 0.1, $0.15,0.2,0.3,0.4,0.5,0.6,0.7,0.8$ and 0.9 to mimic twelve different depths of genome sequencing data. Our results showed that the higher the depth of sequencing, the more novel variants were found, and the rate of false-positive variants was increased dramatically when the depth was lower than $\sim 4 X$, especially when the depth was less than $2 \mathrm{X}$. Additionally, the genome coverage of sequencing increased with depth, and a sequencing depth of 10X achieved 99\% genome coverage (Fig. 4) and ensured high-quality genotyping for pigs (Fig. 5c; Fig. 5d; Fig. 7b). According to our findings, a depth of $10 \mathrm{X}$ was 

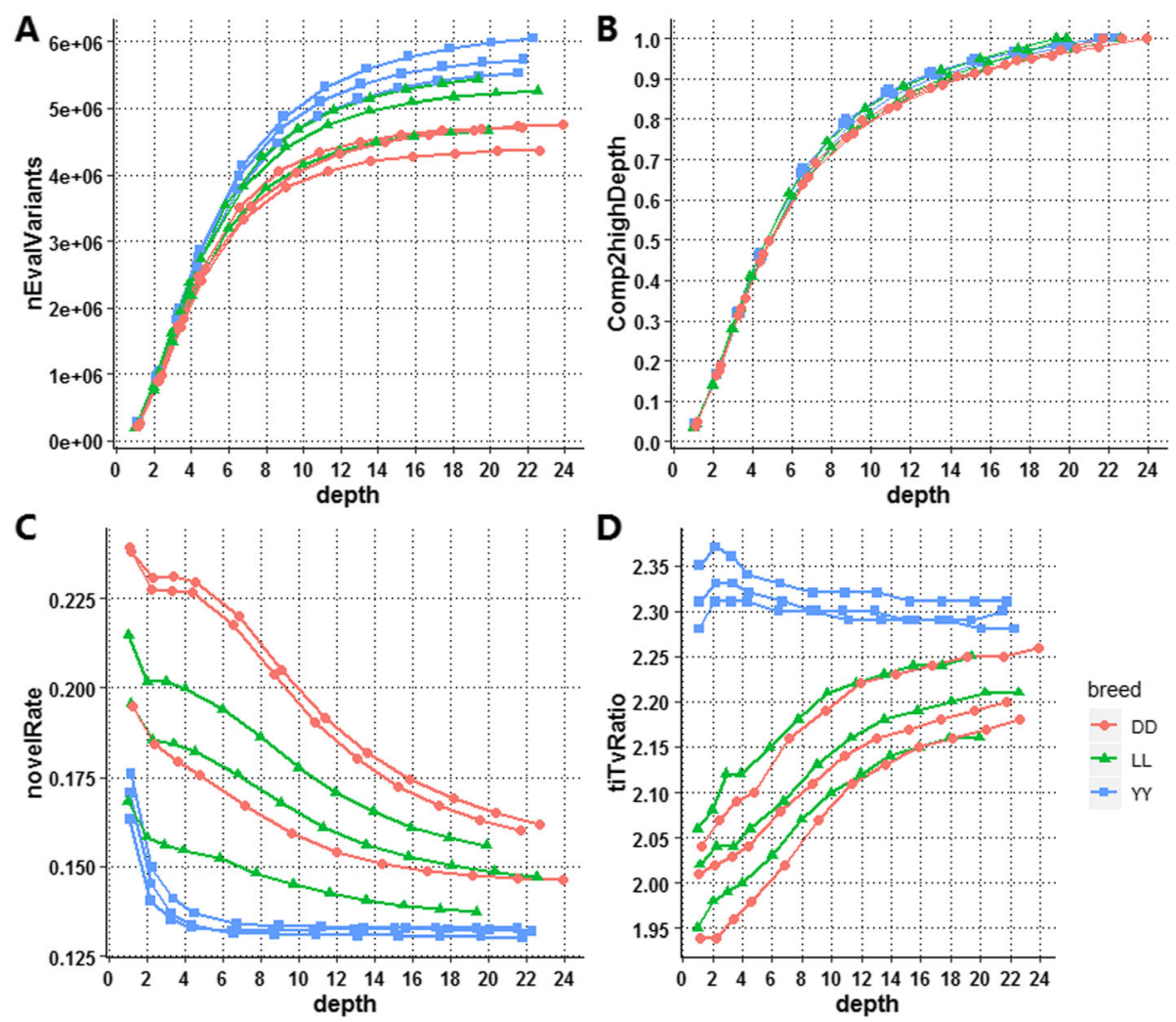

Fig. 5 Discovery power and accuracy of SNP calling. (a) The number of total variants (only SNPs) discovered for each downsampled dataset. (b) Total variant discovery power. We refer to the variants associated with the greatest depth as true variants for each sample and calculate the discovery power for each subdepth, which is the proportion of variants discovered at each depth among the total variants at the greatest depth for each sample. (c) Novel rate. The proportion of variants present in the dbSNP database among the total variants. (d) Ti/Tv ratio. The proportion of the variants observed as transitions (between purines or between pyrimidines) to transversions (between purines and pyrimidines)

not only the point at which saturation was achieved for the function of coverage with depth (Fig. 4) but also the point at which the increase ratio for the total variants discovered slowed (Fig. 5b) and a plateau of discordance with the beadchip data was observed for multisample calling (Fig. 7b). More than $95 \%$ of the genome was covered at $4.35 \mathrm{X}$, which is consistent with other investigations. Rashkin et al. [12] reported that 5-10X was a sufficient sequencing depth to detect common variants and identify associations for a fixed sequencing capacity in simulated data and human datasets. Although sequencing with greater depth can offer more information, many studies have indicated that low-coverage sequencing of large samples is much more cost-effective and powerful than the deep sequencing of fewer individuals. Li et al. [17] reported that when the frequency of variants is greater than $0.2 \%$, only approximately $20 \%$ of the effort is needed to sequence 3000 individuals at $4 \mathrm{X}$ depth while achieving similar power to the sequencing of $>2000$ individuals at $30 \mathrm{X}$ depth. Additionally, Keel's research showed that sequencing a large number of individuals at 4-6X provides higher power than sequencing a smaller number of individuals at a great depth for rare variant detection [35]. The sequencing cost of the latter approach is much higher than that of the former. Therefore, considering the sequencing cost and convenience, a $4 \mathrm{X}$ depth is necessary to achieve more accurate genotyping of pigs since the number of falsepositive variants increases dramatically when the depth is less than $4 \mathrm{X}$. According to the related literature, $4 \mathrm{X}$ is also the depth used in the 1000 Genomes Project for the discovery of disease-associated variants associated with complex diseases in humans [36], and a 10X or greater depth has been used for the assessment of genome-wide genetic variation $[37,38]$ in a pig population or the detection of selection signatures $[39,40]$. In general, $4 \mathrm{X}$ is an appropriate depth for genome-wide association studies, and 10X is an appropriate depth for accurate genotyping and population genetic studies.

To evaluate variant quality for each downsampled genome, the criteria of the novel rate, $\mathrm{Ti} / \mathrm{Tv}$ ratio, and genotype concordance to the greatest depth (21.75X) were investigated. The novel rate gave us a general idea of the 




Fig. 6 Common sites among Beadchip data. The common sites between each depth and beadchip dataset for single-sample calling (s-calling) and multisample calling (m-calling-all, $m$-calling-Alt). The number of common SNPs among the beadchip data (s-calling) was the average value for all three Yorkshire pigs for each depth. The multisample calling of all sites or only the sites with at least one nonreference allele is represented as m-calling-all and m-calling-Alt, respectively

accuracy of variant calling and genotyping. The $\mathrm{Ti} / \mathrm{Tv}$ ratio is an important criterion for assessing the quality of SNP calling [27], which is expected to be 2.1 2.2 for whole-genome variants [41]. Furthermore, a higher Ti/ Tv ratio usually indicates higher accuracy of SNP calling
$[31,41,42]$. Our results regarding the $\mathrm{Ti} / \mathrm{Tv}$ ratio were in agreement with the expected ratio. Compared with Duroc and Landrace, Yorkshire presented a higher $\mathrm{Ti} /$ Tv. Low-coverage sequencing always introduces falsepositive variants in NGS data analysis, but how low this
A

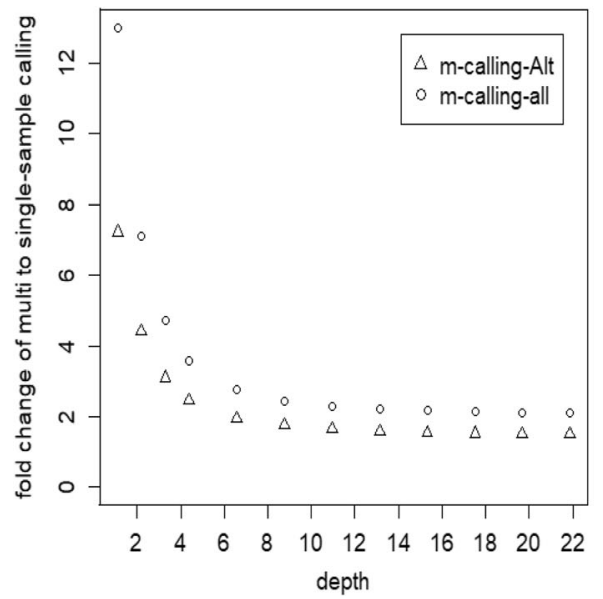

B

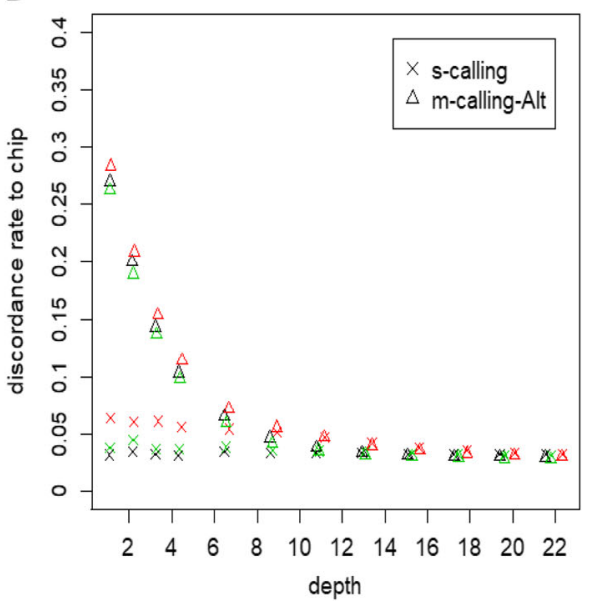

Fig. 7 Fold change between multisample and single-sample calling and the quality of variants. (a) Fold change between multisample and singlesample calling. The ratio of common variants detected in multisample calling to that in single-sample calling. The statistics for multisample calling of all sites ( $m$-calling-all) or only the sites with at least one nonreference allele (m-calling-Alt) are indicated separately. (b) Discordance rate with Beadchip data. Single-sample and multisample calling was compared to PorcineSNP80K BeadChip data for each Yorkshire subsample. For the comparison between single-sample calling and multisample calling, only the common sites of the samples between the PorcineSNP80K BeadChip and whole-genome sequencing SNPs that contained at least one nonreference allele were calculated 
coverage is remains unclear. Our results showed that the false-positive rate was increased significantly when the sequencing depth was less than $\sim 4 \mathrm{X}$ (Fig. 4c; Fig. 5), while the novel rate was extremely high, and the $\mathrm{Ti} / \mathrm{Tv}$ ratio and the concordance rate were also low, indicating that resequencing at depths lower than $4 \mathrm{X}$ could provide inaccurate variants. Moreover, the novel rate increased sharply when the depth was greater than 2.18X (Fig. 4c), which indicated that more false-positive variants were discovered when the depth was less than $\sim 2 \mathrm{X}$. With further decreases in sequencing depth, $2 \mathrm{X}$ is the lower boundary to ensure the quality and coverage of sequencing. This conclusion is in agreement with the simulation study by Fumagalli [23] showing that $2 \mathrm{X}$ is the minimum sequencing depth for obtaining accurate estimates of allele frequencies and identifying polymorphic sites. For the comparison of SNP calling across the three pig breeds, the coverage and concordance with the greatest depth as a function of depth showed no difference across breeds; however, the novel rate and $\mathrm{Ti} / \mathrm{Tv}$ ratio as a function of depth differed between Yorkshire and the other two breeds (Duroc and Landrace). According to B. N. Keel et al. [41], the average Ti/Tv ratios were 2.183, 2.206 and 2.243 for 12 Duroc, 12 Landrace and 48 Yorkshire-Landrace composite sows based on Illumina HiSeq 2500 technology and alignment to the Sscrofa10.2 reference genome, resulting in a mean of 6.1-fold coverage per genome. In addition, the $\mathrm{Ti} / \mathrm{Tv}$ ratio varies greatly by genome region and function [31]; the $\mathrm{Ti} / \mathrm{Tv}$ ratio is generally approximately 3.0 for exome sequencing data and approximately 2.0 outside of exome regions [43]. To our knowledge, it is most likely that biological factors lead to the differences in the Ti/Tv ratio across breeds.

Genotype imputation is widely used in whole-genome association studies and genomic prediction/selection, where a number of individuals are sequenced at a great depth as a reference panel, after which the imputation of individuals on the basis of SNP chip data or low-depth sequencing would be cost efficient. Sequencing data are becoming increasingly important for purposes such as association studies, genomic selection, etc., in which large samples are essential. Thus, to balance sequencing cost and efficiency, the sequencing strategy should be taken into account in practice. Two-stage sequencing has been suggested as a strategy in which some portion of a sample is first sequenced at high coverage as a reference panel, after which the larger sample is sequenced at low coverage, which has proved to be powerful, effective and practical approach [18]. Moreover, STITCH [44], which is a method that was developed for the imputation of genotypes based on sequencing data without the use of additional reference panel or array data, achieves a high imputation accuracy for ultralow-coverage sequencing. The approach resulted in accuracy values of 0.948 and 0.922 for sequencing data for outbred mice (0.15X) and Han Chinese people (1.7X), respectively. Furthermore, GeneImp [45] was developed for the imputation of ultralow-coverage sequencing data $(<1 \mathrm{X})$ with a reference panel, which achieved an even higher accuracy of 0.9. With the development of algorithms and software for low-coverage sequencing or even ultralow-coverage sequencing, additional applications of low-coverage sequencing may be developed, and our research can provide basic guidance for such applications.

In this study, we also compared single-sample calling and multisample calling algorithms. The single-sample calling algorithms were simple, making use solely of reads collected at a single genome position for that sample. However, the multisample calling algorithm included all sample information for a single site. According to our results, multisample calling revealed more variants than single-sample calling, and the lower depth of sequencing, the greater the difference was, with 13fold and two-fold differences in the numbers of variants discovered via multisample calling compared to singlesample calling when sequencing was performed at $1 \mathrm{X}$ and 22X, respectively. Additionally, multisample calling produced more false-positive variants than single-sample calling when the depth was less than 10X. Similar results were found in Bizon's research [46] and Liu's research [47] conducted in a Native American population and another human dataset, respectively. Our results further confirmed the marginally lower nonreference discrepancy value observed for identified single-sample variants than variants obtained via the multi-sample method in sequence data from 65 cattle [48]. Our results suggested that stricter quality control parameters should be implemented in multisample calling, especially when the depth is less than 10X.

\section{Conclusion}

In this study, we explored the relationship between sequencing depth and whole-genome coverage, discovery power, and the accuracy of SNP calling across three pig breeds, Duroc, Landrace and Yorkshire. The genotyping accuracy of the sequencing data was validated with PorcineSNP80K BeadChip data for Yorkshire pigs as well. In addition, multisample and single-sample strategies for SNP calling were compared. Our results showed that a depth of 10X was the point at which saturation was reached for the function of coverage, covering $99 \%$ of the whole pig genome, accounting for $84.42 \%$ of the variants obtained from the deepest genome coverage (21.75X in this study), ensuring good quality of variants from the aspects of the novel rate, Ti/Tv ratio, and beadchip validation. Additionally, more false-positive variants were detected when the depth was less than $4 \mathrm{X}$, 
suggesting that $4 \mathrm{X}$ is the low boundary for reasonable sequencing quality. Compared to single-sample calling, multisample calling was more sensitive, especially at lower depths, and more false-positive variants were detected as well; stricter quality control parameters should be implemented in multisample calling.

\section{Abbreviations}

GATK: The Genome Analysis Toolkit; LoF: Loss-of-function; NGS: Next Generation Sequencing; ORF: Open Reading Frame; SNP: Single Nucleotide Polymorphism; Ti/Tv ratio: Transition/Transversion ratio

\section{Acknowledgements}

Not applicable.

\section{Authors' contributions}

XDD and QZ contributed to the design of the study. YFJ performed the majority of the analysis with contributions from SW. YJ performed the sampling and experiments. XDD and YFJ wrote the manuscript. All authors read and approved the final manuscript.

\section{Funding}

This work was funded by grants from the earmarked fund for China Agriculture Research Systems (CARS-35) and the National Natural Science Foundation of China (31671327), which covered the costs of library preparation and sequencing in this study. Sample collection was funded by the Major Project of Selection for New Livestock and Poultry Breeds of Zhejiang Province (2016C02054-5) and the Anhui Science and Technology Key Project (Grant No. 17030701008). The Program for Changjiang Scholars and Innovation Research Teams in Universities (IRT_15R621) covered the publication cost of this paper and provided support for YFJ and YJ.

\section{Availability of data and materials}

The whole-genome sequencing data for Yorkshire boars obtained in the current study are available from the corresponding author upon reasonable request. The datasets for Duroc and Landrace pigs are available in the NCBI Sequence Read Archive under accession PRJNA343658.

\section{Ethics approval}

Necessary permission was obtained from the owner of the farm for collecting the samples and using in the next study. No benefit interests between owner and the authors. All animal management and sample collection procedures strictly followed the protocol approved by the Institutional Animal Care and Use Ethics Committee (IACUC) at the China Agriculture University. And the IACUC of the China Agricultural University specifically approved this study (permit number DK996).

\section{Consent for publication}

Not applicable.

\section{Competing interests}

The authors declare that they have no competing interests.

\section{Author details}

${ }^{1}$ National Engineering Laboratory for Animal Breeding, Laboratory of Animal Genetics, Breeding and Reproduction, Ministry of Agriculture, College of Animal Science and Technology, China Agricultural University, Beijing 100193, China. ${ }^{2}$ Shandong Provincial Key Laboratory of Animal Biotechnology and Disease Control and Prevention, College of Animal Science and Technology, Shandong Agricultural University, Taian 271001, China.

Received: 25 April 2019 Accepted: 16 October 2019

Published online: 08 November 2019

\section{References}

1. Oetting WS. Exome and genome analysis as a tool for disease identification and treatment: the 2011 human genome variation society scientific meeting. Hum Mutat. 2012;33(3):586-90.
2. Ai H, Yang B, Li J, Xie X, Chen H, Ren J. Population history and genomic signatures for high-altitude adaptation in Tibetan pigs. BMC Genomics. 2014;15(1):834.

3. Li M, Tian S, Yeung CK, Meng X, Tang Q, Niu L, Wang X, Jin L, Ma J, Long K. Whole-genome sequencing of Berkshire (European native pig) provides insights into its origin and domestication. Sci Rep. 2014;4(4):4678.

4. Ai H, Fang X, Yang B, Huang Z, Chen H, Mao L, Zhang F, Zhang L, Cui L, He $W$. Adaptation and possible ancient interspecies introgression in pigs identified by whole-genome sequencing. Nat Genet. 2015;47(3):217-25.

5. Wang MS, Zhang RW, Su LY, Li Y, Peng MS, Liu HQ, Zeng L, Irwin DM, Du $J$, Yao YG. Positive selection rather than relaxation of functional constraint drives the evolution of vision during chicken domestication. Cell Res. 2016;26(5):556.

6. Smith DP, Peay KG. Sequence depth, not PCR replication, improves ecological inference from next generation DNA sequencing. PLoS One. 2014;9(2):e90234.

7. Bhatia D, Wing RA, Yu Y, Chougule K, Kudrna D, Lee S, Rang A, Singh K. Genotyping by sequencing of rice interspecific backcross inbred lines identifies QTLs for grain weight and grain length. Euphytica. 2018;214(2):41.

8. Xing K, Zhu F, Zhai LW, Chen SK, Tan Z, Sun YY, Hou ZC, Wang CD. Identification of genes for controlling swine adipose deposition by integrating transcriptome, whole-genome resequencing, and quantitative trait loci data. Sci Rep. 2016;6:23219.

9. Sims D, Sudbery I, llott NE, Heger A, Ponting CP. Sequencing depth and coverage: key considerations in genomic analyses. Nat Rev Genet. 2014; 15(2):121-32.

10. Desai A, Marwah VS, Yadav A, Jha V, Dhaygude K, Bangar U, Kulkarni V, Jere A. Identification of optimum sequencing depth especially for De novo genome assembly of small genomes using next generation sequencing data. PLoS One. 2013;8(4):e60204.

11. Wang Y, Ghaffari N, Johnson CD, Braganeto UM, Wang H, Chen R, Zhou H: Evaluation of the coverage and depth of transcriptome by RNA-Seq in chickens. Bmc Bioinformatics 2011, 12(Suppl 10):S5-S5.

12. Rashkin S, Jun G, Chen S, Abecasis GR. Optimal sequencing strategies for identifying disease-associated singletons. PLoS Genet. 2017;13(6): e1006811.

13. Ajay SS, Parker SCJ, Abaan HO, Fajardo KVF, Margulies EH. Accurate and comprehensive sequencing of personal genomes. Genome Res. 2011;21(9): 1498-505.

14. Francis WR, Christianson LM, Kiko R, Powers ML, Shaner NC, Haddock SH: A comparison across non-model animals suggests an optimal sequencing depth for de novo transcriptome assembly. BMC Genomics 2013, 14(1):167-167.

15. Chow KS, Ghazali AK, Hoh CC, Mohdzainuddin Z. RNA sequencing read depth requirement for optimal transcriptome coverage in Hevea brasiliensis. Bmc Res Notes. 2014;7(1):69.

16. Liu T, Tsai CH, Lee WB, Chiang JH. Optimizing information in nextgeneration-sequencing (NGS) reads for improving De novo genome assembly. PLoS One. 2013;8(7):e69503.

17. Li Y, Sidore C, Kang HM, Boehnke M, Abecasis GR. Low-coverage sequencing: implications for design of complex trait association studies. Genome Res. 2011;21(6):940-51.

18. Xu C, Wu K, Zhang JG, Shen H, Deng HW. Low-, high-coverage, and twostage DNA sequencing in the design of the genetic association study. Genet Epidemiol. 2016;41:(3).

19. Fang H, Wu Y, Narzisi G, Orawe JA, Barrón LTJ, Rosenbaum J, Ronemus M, Iossifov I, Schatz MC, Lyon GJ. Reducing INDEL calling errors in whole genome and exome sequencing data. Genome Med. 2014;6(10):89.

20. Medvedev P, Fiume M, Dzamba M, Smith T, Brudno M. Detecting copy number variation with mated short reads. Genome Res. 2010;20(11):1613-22.

21. Alves JM, Posada D. Sensitivity to sequencing depth in single-cell cancer genomics. Genome Medicine. 2018;10(1):29.

22. Rizzetto S, Eltahla AA, Lin P, Bull R, Lloyd AR, Ho J, Venturi V, Luciani F. Impact of sequencing depth and read length on single cell RNA sequencing data of T cells. Sci Rep. 2017;7(1):12781.

23. Fumagalli M. Assessing the effect of sequencing depth and sample size in population genetics inferences. PLoS One. 2013;8(11):e79667.

24. Patel RK, Jain M. NGS QC toolkit: a toolkit for quality control of next generation sequencing data. PLoS One. 2012;7(2):e30619.

25. Li H, Durbin R. Fast and accurate short read alignment with burrowswheeler transform: Oxford University press; 2009. 
26. Mckenna A, Hanna M, Banks E, Sivachenko A, Cibulskis K, Kernytsky A, Garimella K, Altshuler D, Gabriel S, Daly M. The genome analysis toolkit: a MapReduce framework for analyzing next-generation DNA sequencing data. Genome Res. 2010;20(9):1297-303.

27. Depristo MA, Banks E, Poplin R, Garimella KV, Maguire JR, Hartl C, Philippakis AA, Del AG, Rivas MA, Hanna M. A framework for variation discovery and genotyping using next-generation DNA sequencing data. Nat Genet. 2011;43(5):491-8.

28. Jian Y, Beben B, Mcevoy BP, Scott G, Henders AK, Nyholt DR, Madden PA, Heath AC, Martin NG, Montgomery GW. Common SNPs explain a large proportion of the heritability for human height. Nat Genet. 2010;42(7):565-9.

29. Price AL, Patterson NJ, Plenge RM, Weinblatt ME, Shadick NA, David R. Principal components analysis corrects for stratification in genome-wide association studies; 2006

30. Wang K, Li M, Hakonarson H. ANNOVAR: functional annotation of genetic variants from high-throughput sequencing data. Nucleic Acids Res. 2010;38(16):e164.

31. Wang J, Raskin L, Samuels DC, Shyr Y, Guo Y. Genome measures used for quality control are dependent on gene function and ancestry. Bioinformatics. 2015;31(3):318.

32. Wang G, Peng B, Leal $S$. Variant association tools for quality control and analysis of large-scale sequence and genotyping Array data. Am J Hum Genet. 2014;94(5):770-83.

33. Team RDC: $R$ : A language and environment for statistical computing. $R$ Foundation for statistical computing, Vienna, Austria. Computing. 2011;14:12-21.

34. Chang CC, Chow CC, Tellier LC, Vattikuti S, Purcell SM, Lee JJ: Secondgeneration PLINK: rising to the challenge of larger and richer datasets. GigaScience,4,1(2015-02-25) 2015, 4(1):7.

35. Le SQ, Durbin R. SNP detection and genotyping from low-coverage sequencing data on multiple diploid samples. Genome Res. 2011;21(6):952-60.

36. Abecasis GR, Adam A, Brooks LD, Depristo MA, Durbin RM, Handsaker RE, Hyun Min K, Marth GT, Mcvean GA. An integrated map of genetic variation from 1,092 human genomes. Nature. 2012;491(7422):56-65.

37. Wang Z, Chen Q, Liao R, Zhang Z, Zhang X, Liu X, Zhu M, Zhang W, Xue M, Yang $\mathrm{H}$. Genome-wide genetic variation discovery in Chinese Taihu pig breeds using next generation sequencing. Anim Genet. 2017:48(1):38-47.

38. Choi J-W, Chung W-H, Lee K-T, Cho E-S, Lee S-W, Choi B-H, Lee S-H, Lim W, Lim D, Lee Y-G, et al. Whole-genome resequencing analyses of five pig breeds, including Korean wild and native, and three European origin breeds. DNA Res. 2015;22(4):259-67.

39. Kim H, Caetanoanolles K, Seo M, Kwon YJ, Cho S, Seo K, Kim H. Prediction of genes related to positive selection using whole-genome Resequencing in three commercial pig breeds. Genomics \& Informatics. 2015;13(4):137.

40. Carl-Johan R, Hendrik-Jan M, Alvaro MB, Khurram M, Shumaila S, Doreen S, Chao W, ?Rjan C, Patric J, Jørgensen CB: Strong signatures of selection in the domestic pig genome. Proc Natl Acad Sci U S A 2012, 109(48):19529-19536.

41. Keel BN, Nonneman DJ, Rohrer GA. A survey of single nucleotide polymorphisms identified from whole-genome sequencing and their functional effect in the porcine genome. Anim Genet. 2017;48:(4).

42. Baes CF, Dolezal MA, Koltes JE, Bapst B, Fritz-Waters E, Jansen S, Flury C, Signer-Hasler $\mathrm{H}$, Stricker C, Fernando R, et al. Evaluation of variant identification methods for whole genome sequencing data in dairy cattle. BMC Genomics. 2014;15(1):948.

43. Bainbridge MN. Targeted enrichment beyond the consensus coding DNA sequence exome reveals exons with higher variant densities. Genome Biol. 2011;12(7):R68

44. Davies RW, Flint J, Myers S, Mott R. Rapid genotype imputation from sequence without reference panels. Nat Genet. 2016;48(8):965-9.

45. Spiliopoulou A, Colombo M, Orchard P, Agakov F, McKeigue P. Genelmp: fast imputation to large reference panels using genotype likelihoods from ultralow coverage sequencing. Genetics. 2017;206(1):91.

46. Bizon C, Spiegel M, Chasse SA, Gizer IR, Li Y, Malc EP, Mieczkowski PA, Sailsbery $\mathrm{JK}$, Wang X, Ehlers CL. Variant calling in low-coverage whole genome sequencing of a native American population sample. BMC Genomics. 2014;15(1):85.

47. Liu X, Han S, Wang Z, Gelernter J, Yang BZ. Variant callers for next-generation sequencing data: a comparison study. PLoS One. 2013;8(9):e75619.

48. Baes CF, Dolezal MA, Koltes JE, Bapst B, Fritzwaters E, Jansen S, Flury C, Signerhasler $\mathrm{H}$, Stricker C, Fernando R. Evaluation of variant identification methods for whole genome sequencing data in dairy cattle. BMC Genomics. 2014;15(1):1-18.

\section{Publisher's Note}

Springer Nature remains neutral with regard to jurisdictional claims in published maps and institutional affiliations.

Ready to submit your research? Choose BMC and benefit from:

- fast, convenient online submission

- thorough peer review by experienced researchers in your field

- rapid publication on acceptance

- support for research data, including large and complex data types

- gold Open Access which fosters wider collaboration and increased citations

- maximum visibility for your research: over $100 \mathrm{M}$ website views per year

At BMC, research is always in progress.

Learn more biomedcentral.com/submissions 